\title{
Aplastic anemia in China
}

\author{
Chunyan Liu, Zonghong Shao \\ Department of Hematology, General Hospital of Tianjin Medical University, Tianjin 300052, China
}

\section{ABSTRACT}

Aplastic anemia (AA) is a hematologic disease characterized by pancytopenia. Up to now, severe aplastic anemia (SAA) has been recognized by international and domestic scholars as an autoimmune disease with bone marrow (BM) failure mediated by the hyperfunctional $\mathrm{T}$ lymphocytes. The incidence of AA is more in China compared with other countries. In the recent years, both the pathogenesis and treatment of $A A$ have made a great progress in our country. Thus, the therapeutic effect of AA was much better than before. Here, we conclude the researches of $A A$ in China.

Key words: aplastic anemia, hematologic disease, T cell, China

\section{INTRODUCTION}

In the recent years, our studies have demonstrated that $T$ cells were hyperfunctional in patients with acquired aplastic anemia (AA). The excessive apoptosis of hematopoietic stem cells caused by cytotoxic $\mathrm{T}$ cells and/ or lymphokines is the main pathogenesis of acquired AA. Up to now, acquired AA has been recognized by some scholars as an autoimmune disease with bone marrow (BM) as target organ attacked by the activated $\mathrm{T}$ lymphocytes. Therefore, immunosuppressive therapy (IST) has become the best choice for the treatment of acquired $\mathrm{AA}$ if there is no sibling donor.

\section{EPIDEMIOLOGY}

Dr. Zonghong Shao, MD, Department of Hematology, General Hospital, Tianjin Medical University, 154 Anshandao, Heping District, Tianjin 300052, China E-mail: shaozonghong@sina.com

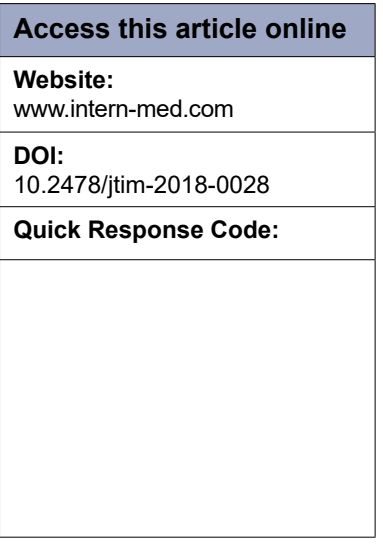

microenvironment, hematopoietic stem cell/progenitor cell deficiencies, and immunity disorders. Shao detected the CD34 positive BM cells of patients with AA and healthy controls and cultured in vitro for CFU-GM, BFU-E, and CFU-E. Finally, they found that the BM CD34 positive cells from patients with AA appear to be normal in percentages and in vitro proliferation/ differentiation capacities. Thus, there may be no deficiencies in the hematopoietic stem cell/progenitor cells of patients with AA ${ }^{[1]}$ With the deepening of research, more and more evidences suggested that AA is a immune-regulated disease characterized by severe pancytopenia and BM failure, caused by destruction of hematopoietic cells by the antigen-specific $\mathrm{T}$ lymphocytes. ${ }^{[2]}$ Our previous studies have demonstrated that suppressor $\mathrm{T}$ lymphocytes (mainly $\mathrm{CD} 8^{+} \mathrm{T}$ cells) have a dramatic increase and hyperfunction in the majority of patients with AA. Those activated $\mathrm{T}$ lymphocytes have obvious inhibitory effect on the growth of BM cells in in vitro experiments. Meanwhile, the quantity of activated effector $\mathrm{T}$ cells $\left(\mathrm{CD} 8^{+}\right.$HLA-DR ${ }^{+}$) was elevated in patients with $\mathrm{AA} .{ }^{[3]}$

While after IST [antithymocyte globulin (ATG) + cyclosporine (CSA)], the cytotoxicity of $\mathrm{CD} 8^{+}$HLA-DR ${ }^{+} \mathrm{T}$ cells was reduced and then reached the normal state. Moreover, the expression of perforin, ${ }^{[4]}$ granular enzyme, 
TNF- $\beta$, and FasL, as well as many other hematopoietic negative regulatory factors, were elevated obviously in these effector $\mathrm{T}$ cells in patients with $\mathrm{AA},{ }^{[5]}$ suggesting that hematopoietic stem and progenitor cells might be destroyed through lymphokine-induced apoptosis. ${ }^{[6]}$ Particularly worth mentioning is the expression of linker for activation of $\mathrm{T}$ cells (LAT) in $\mathrm{CD}^{+} \mathrm{T}$ cells was positively associated with the function molecules (perforin and granzyme B) of $\mathrm{CD}^{+} \mathrm{T}$ cells. Dysregulation of LAT expression and activation may contribute to overfunction of T cells and imbalance of Th1/Th2 subsets and thus lead to hematopoiesis failure in severe aplastic anemia (SAA). ${ }^{[7]}$

Recently, Th1/Th2 imbalance in peripheral blood has been found to be closely related to patients with SAA. The imbalance of myeloid dendritic cells ( $\mathrm{mDCs}$ ) subsets might promote Th0 cells to Th1 cells and cause the overfunction of $\mathrm{T}$ lymphocytes and induction of apoptosis of hematopoietic cells in SAA. ${ }^{[8]}$ It is noteworthy that Th1 cells decreased gradually with hematopoietic function recovery, which might contribute to the immunopathogenesis of SAA. Furthermore, both immature and activated mDCs increased in the BM of patients with SAA. ${ }^{[9]}$

Myeloid dendritic cells secrete IL-12, which is the major stimulator of Th0 cells to Th1 cells. Thus, we infer that the pathogenesis of SAA is related to the increased number and enhanced function of $\mathrm{mDCs}$, which might stimulated by unknown antigen. Then the stimulated mDCs lead hyperfunction of Th1 cells and cytotoxic T lymphocytes and thus ultimately led to the apoptosis of hematopoietic cells.

Although understanding of the immune pathogenesis of SAA gradually improved after many years of research, which antigen activated $\mathrm{mDCs}$ and even $\mathrm{T}$ cells was still unclear. Actually, the immune-initiating antigen has become the focus of our further research. Newly, we have investigated the proteome of $\mathrm{mDCs}$ to further explore the possible antigen that leads to immune activation in SAA. Our research have demonstrated that there are changes in protein expression levels in the SAA group, including PKM2, cofflin protein, and the G-6-PD. ${ }^{[10]}$ But the specific mechanism still needs further validation.

Moreover, researches on the heredity of patients with AA have been conducted recently. Patients with SAA have short telomeres and decreased POT1 expression. TNF- $\alpha$ and IFN $-\gamma$ are found at high concentrations in patients with SAA and may be the effectors that trigger apoptosis through POT1 and ATR. ${ }^{[1]]}$ The research conducted doctor Song showed that the patients with AA and abnormal telomere length have no effect after IST. And these patients should be given other treatments at the initial diagnosis. ${ }^{[12]}$

\section{DIAGNOSIS}

Many years ago, diagnosis of AA mainly depends on the morphology of $\mathrm{BM}$, excluding other hematopathologies. AA diagnosed by this method is essentially a symptom of BM failure containing many different diseases, such as myelodysplastic syndrome (MDS) and paroxysmal nocturnal hemoglobinuria (PNH). With the improvement of examination, we gradually distinguished several other diseases with $\mathrm{BM}$ failure from $\mathrm{AA}$ and purified $\mathrm{AA}$ into a kind of independent disease system with relatively clear pathological mechanism. Some MDS was also obvious dysplasia and should be distinguished from AA. We have found that the basic difference in MDS from SAA was malignant clonal proliferation. Cytogenetics maybe helpful and abnormal chromosomes can be considered as an exclusion criterion. Currently, the emergence of new diagnostic methods, namely, CD55 and CD59, made the diagnosis of $\mathrm{PNH}$ more precise, but there are still some PNH called SAA-PNH syndrome that cannot be distinguished from SAA. Actually, if more than $10 \%$ of the PNH clone can be found in the patient with obviously abnormal hematopoiesis and decrease in mature hemocytes, it can be diagnosed as PNH. Meanwhile, we should exclude the fanconi anemia (FA) caused by congenital anomaly and AHA caused by some incentive. In addition, with the long-term research on $\mathrm{AA}$, we purified a new disease from AA, and we called it immuno-related hemocytopenia (IRP), which was characterized by hyperfunction of B lymphocyte. ${ }^{[13]}$ In the recent years, we observed that these patients responded well to corticosteroid and high-dose intravenous immunoglobulin treatment, indicating cytopenia might be mediated by autoantibodies. We retrospectively analyzed 166 patients with idiopathic cytopenia of undetermined significance (ICUS), some of which were detected as autoantibodies on BM hematopoietic cells BM mononuclear cell (BMMNC)-Coombs test, flow cytometry, western blot, and immunofluorescence (IF). ${ }^{[14]}$

We found that $25.9 \%(43 / 166)$ of patients had positive BMMNC-Coombs test or FCM analysis and $72.1 \%(31 / 43)$ of which had IgG-autoantibody positive by western blot. More than half of the patients had hyper-BM cellularity with a higher percentage of nucleated erythroid cells in the sternum. Therefore, by our clinical experience, we suggested the following points during the diagnosis of AA. First, take the evidence of hyperfunction in T lymphocyte. Next, different parts of the $\mathrm{BM}$ puncture were required to judge the state of hematopoiesis, especially including sternal BM puncture. Third, BM biopsies, which mainly reflect the structure of the $\mathrm{BM}$ and special ingredients such as fiber and "stone bone," were of similar importance. We should combine the two examinations to evaluate the hyperplasia of BM in patients. ${ }^{[15]}$ 


\section{TREATMENT}

Once AA is diagnosed, we should take treatment measures as soon as possible. For young patients with SAA and HLA-matched sibling donor, hematopoietic stem cell transplantation (HSCT) becomes the preferred treatment for granted. Also in the recent years, the unrelated donor HSCT and haploidentical hematopoietic stem cell transplantation (Haplo-HSCT) were considered as an alternative therapy for SAA if there is no suitable donor for matched sibling donor allogeneic hematopoietic stem cell transplantation (MSD-HSCT) $\cdot{ }^{[16]}$ However, because of the donor, economic, graft-versus-host disease (GVHD), the use of HSCT to treat SAA was restricted. For those patients older than or younger than 40 years with no HLAmatched sibling donor, IST should be the first choice. ${ }^{[17]}$ IST could inhibit the hyperfunction of $\mathrm{T}$ lymphocytes, reduce the negative hematopoietic regulatory factors, and remove the damage of hematopoietic cells. This regimen has become the first-line treatment for AA (especially SAA) and has been included in the guidelines of China and domestic. There was a significant risk of relapse with rapid tapering of cyclosporine, and the treatment should be continued for a long time until the immunoreaction is completely back to normal. It generally takes a small dose (as small as $25 \mathrm{mg}$ ) for several years. Meanwhile, IST often combines with hematopoietic stimulating factor (HGF), thrombopoietin (TPO), and androgen in case to promote hematopoietic function of BM. An adequate course of HGF leads to lower rates of early infection and mortality, shorter duration of cytopenia and blood transfusion dependence, and faster recovery of $\mathrm{BM}$ hematopoiesis. The addition of HGFs to sequential intensive immunosuppressive therapy (SIIST) was well tolerated in all patients. ${ }^{[18]}$ We observed that granulocyte transfusions combining with G-CSF to treat severe infections in patients with SAA increase the response rate of antifungal and antibiotic therapies. The survival rate at 30, 90, and 180 days were $89 \%, 70 \%$, and $66 \%$, respectively, which were longer than that before. ${ }^{[19]}$ Study by doctor Wang showed that the time to platelet and red blood cell transfusion independence was shorter in patients with SAA who received TPO than in those who have not received TPO treatment. ${ }^{[20]}$ Thus the use of TPO could improve hematologic response and promote BM recovery in patients with SAA receiving IST. Androgen can promote hematopoiesis by stimulating the secretion of erythropoietin (EPO) of kidney and increasing the sensitivity of immature red cell to the EPO. Before the ATG/CSA, androgen has already been used in the treatment of AA; now it has became an aid drug in the treatment of AA. ${ }^{[21]}$ IST is the "fundamental treatment" of $\mathrm{AA}$, and immune support treatment was equally important and helpful. Most patients with AA have extremely low immunity with the lack of granulocyte; powerful immune support therapy can improve the humoral immunity. As symptomatic treatment, appropriate dose of gammaglobulin or thymosin can be used for patients with AA. ${ }^{[22]}$

\section{PROGNOSIS}

SAA occurs urgently and had an extremely high mortality rate earlier. Nearly, a decade, the prognosis of SAA significantly improved as a result of better treatment. ${ }^{[23]}$ The effective rate of sib-HSCT is about $80 \%$, but only $30 \%$ of patients with SAA can obtain the HLA-matched sibling donor. The therapeutic effect of IST also improved in the recent years, almost same as the sib-HSCT. Research by the Beijing Children Hospital on the rabbit ATG showed that the total effect was $77 \%$, and in the study by doctor Cao, it was $83.3 \% .{ }^{[24]}$ In China, the use of horse ATG is limited, but the pig ATG was frequently used because of its less cost. The research of Han summarized the clinical data of 48 patients who received treatment with pig ATG, the total therapeutic effect was $82.2 \%$, almost the same as rabbit ATG. ${ }^{[2]}$ The study of doctor Wei obtained the same result, revealing that the pig ATG was the same in effectiveness compared with that of the rabbit or horse ATG. ${ }^{[2]}$ Meanwhile, the commission AA patients still have the opportunity of relapse and clonal evaluation. Cui reported 7 patients who had monosomy 7 malignant clonal evolution in the Tianjin General Hospital. Ma also reported 19 patients who evoluted to MDS/AML after treated with IST, and the main clonal abnormal was monosomy $7 .{ }^{[2]}$ Only 1 person have the evaluation of plus $8 ; 2$ persons have the abnormal of complicated karyotypes. Research by doctor sun showed that PNH clone are detectable in patients with AA after treatment with IST. ${ }^{[28]}$ And the appearance of PNH clone had not affected the therapeutic efficacy of patients with AA.

General aspect, during the nearly 20 years, not only the pathogenesis and diagnosis but also the treatment of SAA has made a great progress in China. And the guideline of the diagnosis and treatment about AA in China has been published in the Chinese Journal of Hemotology. For the great efforts made by all hematologic doctors in China, the therapeutic effect of AA was better than before. But there are still more questions about the pathogenesis of $\mathrm{AA}$ that have to be explored. The treatment of SAA should also be modified in the future.

\section{Source of Foundation}

This work was supported by the National Natural Science Foundation of China (81870101, 81800119, 81800120, 81400085, 81570106, 81600093, 81570111, and 81400088). 


\section{Conflict of Interest}

The authors declare no competing financial interests.

\section{REFERENCE}

1. Chen $\mathrm{G}$, Shao Z, Jia H. In vitro proliferation and differentiation of bone marrow stem cells of aplastic anemia patients. Zhonghua Xue Ye Xue Za Zhi 1999; 20: 529-31.

2. Shao ZH. Studies on aplastic anemia in China. Basic Clin Med 2007; 27: 233-7.

3. Feng L, Fu R, Wang HQ, Wang J, Liu CY, Li LJ, et al. Effect of CD8+ effector $\mathrm{T}$ cells on the hematopoiesis pathway damage in the patients with severe aplastic anemia. Zhonghua Xue Ye Xue Za Zhi 2011; 32: 597-601.

4. Zhang J, Fu R, Wang J, Li LJ, Song J, Qu W, et al. Perforin gene mutations in patients with acquired severe aplastic anemia. Zhongguo Shi Yan Xue Ye Xue Za Zhi 2011, 19: 431-4.

5. Liu CY, Fu R, Wang J, Liang Y, Qu W, Ruan EB, et al. Expression of lymphokines in CD8(+)HLA-DR(+) T lymphocytes of patients with severe aplastic anemia. Zhonghua Yi Xue Za Zhi 2012, 92: 1240-3.

6. Liu CY, Fu R, Wang HQ, Li LJ, Liu H, Guan J, et al. Fas/FasL in the immune pathogenesis of severe aplastic anemia. Genet Mol Res 2014; 13: 4083-8.

7. Sheng W, Liu C, Fu R, Wang H, Qu W, Ruan E, et al. Abnormalities of quantities and functions of Linker for Activations of T cells (LAT) in severe aplastic anemia. Eur J Haematol 2014; 93: 214-23.

8. Zonghong S, Meifeng T, Huaquan W, Limin X, Jun W, Rong F, et al. Circulating myeloid dendritic cells are increased in individuals with severe aplastic anemia. Int J Hematol 2011; 93: 156-62.

9. Wang J, Shao ZH, Fu R, Ruan EB, Qu W, Liang Y, et al. Study On the dendritic cell subsets in peripheral blood and its relationship with the expressions of T-bet and GATA-3 in lymphocytes in severe aplastic anemia. Zhonghua Xue Ye Xue Za Zhi 2008; 29: 733-6.

10. Liu C, Sheng W, Fu R, Wang H, Li L, Liu H, et al. Differential expression of the proteome of myeloid dendritic cells in severe aplastic anemia. Cell Immunol 2013; 285: 141-8.

11. Wang T, Mei SC, Fu R, Wang HQ, Shao ZH. Expression of Shelterin Component POT1 Is Associated with Decreased Telomere Length and Immunity Condition in Humans with Severe Aplastic Anemia. J Immunol Res 2014; 2014: 439530.

12. Song JY, Kuang LP, Wang Y, Li YH, Wu JL, Zhang H, et al. The relationship of teiomere and telomerase activity with outcome of aplastic anemia after immunesu pysessive therapy. Zhonghua Xue Ye Xue Za Zhi 2013; 34: 771-6.

13. Shao Y, Fu R, Liu H, Wang Y, Ding S, Wang H, et al. IgG autoantibody subclasses altered in immuno-related hemocytopenia. Cell Immunol 2015; 294: 13-20.

14. Liu H, Fu R, Wang Y, Liu H, Li L, Wang H, et al. Detection and analysis of autoantigens targeted by autoantibodies in immunorelated pancytopenia. Clin Dev Immunol 2013; 2013: 297678.

15. Hematology Branch of the Chinese Medical Association RBC disease (anemia) Study Group. Expert consensus on the diagnosis and treatment of aplastic anemia. Zhonghua Xue Ye Xue Za Zhi 2010; 31: 790-2.
16. Zuo S, Xu L. The current status and development of haploidentical allogeneic hematopoietic stem cell transplantation for severe aplastic anemia. Zhonghua Xue Ye Xue Za Zhi 2015; 36: 707-10.

17. Socie G. Allogeneic BM transplantation for the treatment of aplastic anemia: current results and expanding donor possibilities. Hematology Am Soc Hematol Educ Program 2013, 2013: 82-6.

18. He G, Shao Z, Zhang Y, Liu H, Li K, Song L, et al. Sequential intensified immunosuppressive therapy combining with hematopoietic growth factors in the treatment of severe aplastic anemia. Zhonghua Xue Ye Xue Za Zhi 2001; 22: 177-81.

19. Wang H, Wu Y, Fu R, Qu W, Ruan E, Wang G, et al. Granulocyte transfusion combined with granulocyte colony stimulating factor in severe infection patients with severe aplastic anemia: a single center experience from China. PLoS One 2014; 9: e88148.

20. Wang H, Dong Q, Fu R, Qu W, Ruan E, Wang G, et al. Recombinant human thrombopoietin treatment promotes hematopoiesis recovery in patients with severe aplastic anemia receiving immunosuppressive therapy. BioMed Res Int 2015; 2015: 597293.

21. Yang L, Wang H, Shao Z. Advances in the treatment of severe aplastic anemia. Zhonghua Xue Ye Xue Za Zhi 2015; 36: 711-5.

22. Liu CY, Shao ZH. Immune treatment of aplastic anemia. J Clin Int Med 2012; 29: 365-7.

23. Zhou X, Wu RH, Duan YT. Antithymocyte globulin combining cyclosporin in treatment of pediatric severe aplastic anemia. J Appl Clin Pediatr 2007; 22: 1178-80.

24. Su Y, Wu RH, Ma J, Zhou H, Zhang R. Evaluation of the efficacy of immunosuppressive therapy with rabbit-antithymocyte globulin combined with Cyclosporine in children with severe aplastic anemia. J Appl Clin Pediatr 2015; 30: 189-93.

25. Bing H, Siyi Y, Wei Z, Jian L, Minghui D, Li J, et al. The use of anti-human T lymphocyte porcine immunoglobulin and cyclosporine A to treat patients with acquired severe aplastic anemia. Acta Haematol 2010; 124: 245-50.

26. Wei J, Huang Z, Guo J, Zhang Y, Wang C, Zhu X, et al. Porcine antilymphocyte globulin (p-ALG) plus cyclosporine A (CsA) treatment in acquired severe aplastic anemia: a retrospective multicenter analysis. Ann Hematol 2015; 94: 955-62.

27. Cui N, Fu R, Qu W, Ruan E, Wang X, Wang G, et al. Clinical analysis on malignant clonal hematopoiesis in severe aplastic anemia patients with immunosuppressive therapy. Zhonghua Yi Xue Za Zhi 2015; 95: 2620-2.

28. Sun YX, Zhu MQ, He GS, Wang XL, Fang BZ, Lu C, et al. The variation and clinical significance of paroxysmal nocturnal hemoglobinuria clone in patients with aplastic anemia before and after immunosuppressive therapy. Zhonghua Nei Ke Za Zhi 2013; 52: 585-9.

How to cite this article: Liu C, Shao Z. Aplastic anemia in China. J Transl Intern Med 2018; 6: 134-7. 